Hautarzt 2009 - 60:838-838

DOI 10.1007/s00105-009-1851-9

(c) Springer Medizin Verlag 2009

\section{Redaktion}

J. Krutmann, Düsseldorf

Redaktionelle Mitarbeit

J.M. Baron, Aachen

M. Berneburg, Tübingen

R. Gläser, Kiel

C. Hafner, Regensburg

B. Homey, Düsseldorf

\author{
C. Hafner \\ Klinik und Poliklinik für Dermatologie, Universität Regensburg
}

\title{
Oligoklonalität multipler primärer Melanome
}

sie eine LOH (Loss of Heterozygosity)Analyse mittels 26 polymorphen Mikrosatellitenmarkern durch und verglichen das erhaltene genetische Profil zwischen beiden Melanomen eines Patienten. Zusätzlich wurden statistische Analysen verwendet, um die Signifikanz unterschiedlicher LOH-Muster zu errechnen und eine zufällige Konkordanz der genetischen Alterationen auszuschließen. Es zeigte sich, dass bei 17 von 19 Patienten die beiden Melanome eindeutig ein unterschiedliches genetisches Profil aufwiesen und damit als oligoklonal anzusehen sind. Lediglich zwei Patienten zeigten Hinweise, dass es sich bei den beiden untersuchten Melanomen um monoklonale, miteinander verwandte Tumoren handeln könnte. Somit wird die bisherige klinische und histologische Einschätzung, dass es sich bei multiplen syn- oder metachron auftretenden Melanomen in der Regel um unabhängige Primärtumoren handelt, durch diese neuen molekulargenetischen Analysen bestätigt.

\section{Fazit für die Praxis}

Bei Patienten mit multiplen Melanomen, die gleichzeitig oder zeitlich versetzt auftreten, handelt es sich offensichtlich in der weit überwiegenden Zahl der Fälle um genetisch bzw. klonal nicht miteinander verwandte Primärtumoren, die einer entsprechenden operativen Therapie zuzuführen sind. Die signifikant erhöhte Inzidenz von Zweitmelanomen bei Melanompatienten gegenüber Vergleichspersonen erklärt sich damit in erster Linie durch die ge- netische Disposition sowie die individuelle Exposition gegenüber Risikofaktoren aus der Umwelt (z.B. UV-Licht), nicht jedoch durch eine Absiedlung von Melanomzellen aus dem initial aufgetretenen Melanom.

\section{Korrespondenzadresse: \\ PD Dr. Christian Hafner \\ Klinik und Poliklinik für Dermatologie \\ Universität Regensburg \\ Franz-Josef-Strauss-Allee 11 \\ 93053 Regensburg \\ christian.hafner@klinik.uni-regensburg.de}

Interessenonflikt. Der korrespondierende Autor gibt an, dass kein Interessenkonflikt besteht.

\section{Literatur}

1. Orlow I, Tommasi DV, Bloom B et al (2009) Evaluation of the clonal origin of multiple primary melanomas using molecular profiling. J Invest Dermatol 129:972-82
Orlow et al. [1] untersuchten daher den klonalen Charakter von jeweils zwei synoder metachron aufgetretenen Melanomen bei insgesamt 19 Patienten, welche klinisch als unabhängige Primärtumoren eingestuft worden waren. Hierzu führten 